“(C) 2015 IEEE. Personal use of this material is permitted. Permission from IEEE must be obtained for all other uses, in any current or future media, including reprinting/republishing this material for advertising or promotional purposes, creating new collective works, for resale or redistribution to servers or lists, or reuse of any copyrighted component of this work in other works." 


\section{Unitary Differential Space-Time-Frequency Codes for MB-OFDM UWB}

\author{
L. C. Tran \\ University of Wollongong \\ Wollongong, Australia
}

\author{
A. Mertins \\ University of Luebeck \\ Luebeck, Germany
}

\author{
E. Dutkiewicz \\ University of Macquarie \\ Sydney, Australia
}

\author{
X. Huang \\ CSIRO \\ Marsfield, Australia
}

\begin{abstract}
In a multiple-input multiple-output (MIMO) multiband orthogonal frequency division multiplexing (MB-OFDM) ultra-wideband (UWB) system, coherent detection where the channel state information (CSI) is assumed to be exactly known at the receiver requires the transmission of a large number of symbols for channel estimation, thus reducing the bandwidth efficiency. This paper examines the use of unitary differential space-time frequency codes (DSTFCs) in MB-OFDM UWB, which increases the system bandwidth efficiency due to the fact that no CSI is required for differential detection. The proposed DSTFC MB-OFDM system would be useful when the transmission of multiple channel estimation symbols is impractical or uneconomical. Simulation results show that the application of DSTFCs can significantly improve the bit error performance of conventional differential MB-OFDM system (without MIMO).
\end{abstract}

Index Terms-UWB, MB-OFDM, DSTFC, STFC, MIMO.

\section{INTRODUCTION}

Combination of the emerging technologies multi-band orthogonal frequency division multiplexing ultra-wideband (MB-OFDM UWB) [1], multiple-input multiple-output (MIMO), and space-time-frequency codes (STFCs) may provide a significant improvement in the form of maximum achievable communication range, bit error performance, system capacity, data rate, or a combined form of those. The combination of MB-OFDM UWB, MIMO and STFCs to which we will refer as STFC MB-OFDM UWB systems has been considerably examined in the literature, such as [2], [3], [4], [5], [6], [7], [8].

In all aforementioned works, channel state information (CSI) is assumed to be known exactly at the receiver, thus allowing the receiver to perform coherent detection. According to [1], six MB-OFDM symbols are transmitted in the physical layer convergence protocol (PLCP) preamble for channel estimation between each pair of Tx and Rx antennas, thus facilitating coherent detection at the receiver. In a MIMO system consisting of $M$ Tx and $N \mathrm{Rx}$ antennas, the required number of symbols for this purpose might be as large as $6 M N$, except for the case where superimposed training techniques, such as in [9], [10], [11], are used to reduce the number of channel estimation symbols transmitted within the preamble. Therefore, transmission of large number of MB-OFDM symbols for channel estimation reduces significantly the system bandwidth efficiency. In fast fading channels or in very high data rate systems, transmission of large number of MB-OFDM symbols for channel estimation is a hassling task and might even be impractical or uneconomical. In these cases, noncoherent detection (or differential detection), where no CSI is required for decoding signals at the receiver, would be the best candidate.

For differential transmission in general OFDM systems associated with a MIMO model, various techniques have been proposed in the literature, such as [12], [13], [14], [15], [16] and [17]. However, differential transmission in MB-OFDM systems associated with MIMO has not been considered yet. There are two main differences between channel characteristics in conventional OFDM systems and in MB-OFDM UWB ones. Firstly, channels in the latter are much more dispersive than those in the former, with the average number of multipaths in some channel models reaching some thousands [18]. Secondly, channel coefficients in the former are usually considered to be Rayleigh distributed, while those in the latter are log-normally distributed [18]. These differences of MBOFDM systems require simulations to be run with the special channel model that has been proposed in [18]. This will be mentioned in detail later in the simulation section of this paper.

Besides the different channel model, a MB-OFDM system differs from a general OFDM one in the fact that the former transmits consecutive MB-OFDM symbols at different frequency sub-bands thanks to the application of different TimeFrequency Codes (TFCs) [1, p.60]. This technique should also be taken into account in the proposal of the differential space-time-frequency codes (DSTFCs) MB-OFDM system. This issue will be discussed later in Section III of this paper.

For the above reasons, the systems incorporating MBOFDM UWB, MIMO and differential transmission must be more specifically analyzed, though there exist several similarities between those systems and the systems incorporating conventional OFDM, MIMO and differential transmission. Thus this paper is the first case study that examines the application of DSTFCs in MB-OFDM UWB communications.

The paper is organized as follows. Section II reviews briefly the mathematical model of our proposed STFC MB-OFDM UWB system [4]. In Section III, unitary DSTFCs are proposed for MB-OFDM UWB and decoding metrics are derived for the proposed DSTFCs. Simulation results are mentioned in Section IV and conclusions are derived in Section V.

Notations: The following notations will be used throughout the paper. The superscripts $(.)^{*},(.)^{T}$ and $(.)^{H}$ denote the 

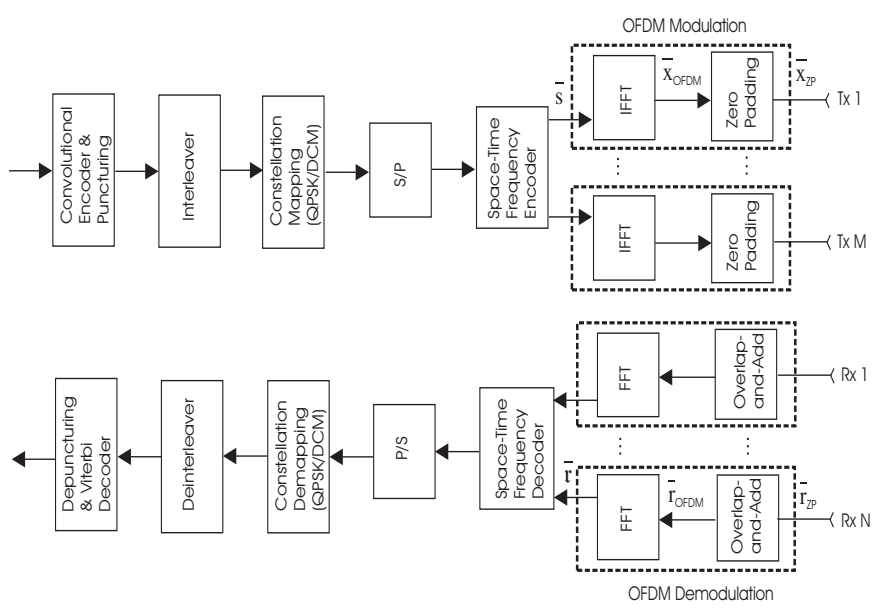

Fig. 1. Structural diagram of the proposed STFC MB-OFDM UWB system [4].

complex conjugation, transposition operation and conjugate transpose operation respectively. We denote $\overline{\mathbf{a}}_{j} \bullet \overline{\mathbf{b}}_{j}, \overline{\mathbf{a}}_{j} \otimes \overline{\mathbf{b}}_{j}$ and $\overline{\mathbf{a}}_{j} * \overline{\mathbf{b}}_{j}$ to be the element-wise (or Hadamard) product, the linear convolution and the cyclic convolution between the two vectors $\overline{\mathbf{a}}_{j}$ and $\overline{\mathbf{b}}_{j}$ respectively. Denote $N_{f f t}$ to be the FFT/IFFT size (for MB-OFDM UWB communications [1], $\left.N_{f f t}=128\right)$. Further, $\Re\{c\}$ and $\Im\{c\}$ denote the real and imaginary parts of the complex number $c$. The notation $\operatorname{diag}\left(\overline{\mathbf{a}}_{j}\right)$ denotes a square diagonal matrix formed by stacking the vector $\overline{\mathbf{a}}_{j}$ on the main diagonal of the matrix, while $\left\{\operatorname{diag}\left(\overline{\mathbf{a}}_{j}\right)\right\}_{M_{j} \times N_{j}}$ denotes a $M_{j} \times N_{j}$-sized rectangular matrix whose elements are diagonal matrices $\operatorname{diag}\left(\overline{\mathbf{a}}_{j}\right)$. We state that the two subsets of indices $\{m, k\}$ and $\{\dot{m}, \hat{k}\}$ to be different, denoted as $\{m, k\} \neq\{m, k\}$, if at least one of the following two inequalities $m \neq \dot{m}$ and $k \neq \dot{k}$ occurs. Finally, we define $\overline{\mathbf{1}}$ as a column vector of length $N_{D}$, whose elements are all 1 .

\section{STFC MB-OFDM UWB SYSTEM}

The proposed STFC MB-OFDM UWB system [4] consisting of $M \mathrm{Tx}$ antennas and $N \mathrm{Rx}$ antennas, with the notations of signals at the considered reference points, is depicted in Fig. 1. The transmitted STFC is denoted as a matrix $\mathbf{S}_{t}=\left\{\overline{\mathbf{s}}_{t, m}\right\}_{T \times M}$, where $T$ denotes the number of MBOFDM symbol time slots required to transmit the whole STFC block. The code matrix $\mathbf{S}$ can be structured in a similar way as orthogonal space-time block codes (OSTBCs) [19], [20], [21] or quasi-orthogonal space-time block codes (QOSTBCs) [22] in conventional wireless STBC MIMO systems, except for that each element $\overline{\mathbf{s}}_{t, m}$ is not a complex number, but is defined as a column vector $\overline{\mathbf{s}}_{t, m}=\left[s_{t, m, 1} s_{t, m, 2} \ldots s_{t, m, N_{f f t}}\right]^{T}$. The vectors $\overline{\mathbf{s}}_{t, m}$ are the original transmitted data before IFFT. The symbols $s_{t, m, k}$, for $k=1, \ldots, N_{f f t}$, are drawn from a PSK, QAM or dual carrier modulation (DCM) [23] signal constellation.

Denote $\mathcal{X}=\left\{\overline{\mathbf{x}}_{O F D M, t, m}\right\}_{T \times M}$ to be the matrix whose elements are the $N_{f f t}$-point IFFTs of the respective elements in $\mathbf{S}_{t}$, then $\mathcal{X}=\left\{I F F T\left\{\overline{\mathbf{s}}_{t, m}\right\}\right\}_{T \times M}=\left\{\overline{\mathbf{x}}_{O F D M, t, m}\right\}_{T \times M}$. The symbols $\overline{\mathbf{x}}_{O F D M, t, m}$ are referred to as MB-OFDM sym- bols. Further, denote $\mathcal{X}_{\mathbf{Z P}}=\left\{\overline{\mathbf{x}}_{Z P, t, m}\right\}_{T \times M}$ to be the matrix whose elements are the respective elements in $\mathcal{X}$ appended by a zero padded suffix (ZPS), which, according to [1], includes 37 zeros. At the transmission of the $t$-th MB-OFDM symbol, we denote $\overline{\mathbf{h}}_{t, m, n}=\left[\begin{array}{ll}h_{t, m, n, 1} & h_{t, m, n, 2} \ldots h_{t, m, n, L_{m, n},}\end{array}\right]^{T}$ to be the channel vector between the $m$-th $\mathrm{Tx}$ and $n$-th $\mathrm{Rx}$ antennas, for $m=1, \ldots, M, n=1, \ldots, N$, where the channel coefficients $h_{m, n, l}$ of the $l$-th path, $l=1, \ldots, L_{m, n}$, in this channel are modeled as independent log-normally distributed random variables (RVs). Let $L_{\max }=\max \left\{L_{m, n}\right\}$, for $m=1, \ldots, M, n=1, \ldots, N$. Denote the MB-OFDM UWB channel coefficient matrix as $\mathbf{H}=\left\{\overline{\mathbf{h}}_{m, n, Z P}\right\}_{M \times N}$ where the vector $\overline{\mathbf{h}}_{m, n, Z P}$ is created from the corresponding channel vector $\overline{\mathbf{h}}_{m, n}$ by adding zeros to have the length $L_{\max }$.

At the transmission of the $t$-th MB-OFDM symbol, the received signal at the $n$-th $\mathrm{Rx}$ antenna is calculated as

$$
\overline{\mathbf{r}}_{Z P, t, n}=\sum_{m=1}^{M}\left(\overline{\mathbf{x}}_{Z P, t, m} \otimes \overline{\mathbf{h}}_{t, m, n}\right)+\overline{\mathbf{n}}_{t, n} .
$$

The elements of noise vector $\overline{\mathbf{n}}_{t, n}$ are considered to be independent complex Gaussian RVs.

In MB-OFDM system, a ZPS of length $N_{Z P S}=37$ [1] is appended to each symbol $\overline{\mathbf{x}}_{O F D M, t, m}$ at the transmitter to create a transmitted symbol $\overline{\mathbf{x}}_{Z P, t, m}$. At the receiver, an overlap-and-add operation (OAAO) must be performed before FFT (i.e. $N_{Z P S}$ samples of a received symbol $\overline{\mathbf{r}}_{Z P, t, n}$ from $\left(N_{f f t}+1\right)$ to $\left(N_{f f t}+N_{Z P S}\right)$ are added to the beginning of that received symbol. Then the first $N_{f f t}$ samples of the resulting symbol will be used to decode the transmitted symbol). As a result, after performing OAAO for the received signal $\overline{\mathbf{r}}_{Z P, t, n}$ in (1) and then taking the first $N_{f f t}$ resulting samples, denoted as $\overline{\mathbf{r}}_{O F D M, t, n}$, the following equation is deduced

$$
\overline{\mathbf{r}}_{O F D M, t, n}=\sum_{m=1}^{M} \overline{\mathbf{x}}_{O F D M, t, m} * \overline{\mathbf{h}}_{t, m, n}+\overline{\mathbf{n}}_{t, n} .
$$

For the circular convolution, we have the following property

$$
\begin{aligned}
\overline{\mathbf{x}}_{O F D M, t, m} * \overline{\mathbf{h}}_{m, n}= & \operatorname{IFFT}\left\{F F T\left\{\overline{\mathbf{x}}_{O F D M, t, m}\right\} \bullet\right. \\
& \left.\operatorname{FFT}\left\{\overline{\mathbf{h}}_{t, m, n}\right\}\right\} \\
= & \operatorname{IFFT}\left\{\overline{\mathbf{s}}_{t, m} \bullet \overline{\mathfrak{h}}_{t, m, n}\right\}
\end{aligned}
$$

where $\overline{\mathfrak{h}}_{t, m, n}$ is the $N_{f f t}$-point FFT of the channel vector $\overline{\mathbf{h}}_{t, m, n}$, i.e. $\overline{\mathfrak{h}}_{t, m, n}=F F T\left\{\overline{\mathbf{h}}_{t, m, n}\right\}$. We denote $\overline{\mathfrak{h}}_{t, m, n}=$ $\left[\hbar_{t, m, n, 1} \hbar_{t, m, n, 2} \ldots \hbar_{t, m, n, N_{f f t}}\right]^{T}$.

After going through the FFT block at the receiver, the received signal becomes

$$
F F T\left\{\overline{\mathbf{r}}_{O F D M, t, n}\right\}=\sum_{m=1}^{M} \overline{\mathbf{s}}_{t, m} \bullet \overline{\mathfrak{h}}_{t, m, n}+F F T\left\{\overline{\mathbf{n}}_{t, n}\right\} .
$$

Denote $\quad \overline{\mathfrak{r}}_{t, n}=\left[\begin{array}{ll}\mathfrak{r}_{t, n, 1} & \mathfrak{r}_{t, n, 2} \ldots \mathfrak{r}_{t, n, N_{f f t}}\end{array}\right]^{T}=$ $F F T\left\{\overline{\mathbf{r}}_{O F D M, t, n}\right\}$ and $\overline{\mathfrak{n}}_{t, n}=\left[\mathfrak{n}_{t, n, 1} \mathfrak{n}_{t, n, 2} \ldots \mathfrak{n}_{t, n, N_{f f t}}\right]^{T}=$ $F F T\left\{\overline{\mathbf{n}}_{t, n}\right\}$. Then (2) can be rewritten as follows

$$
\overline{\mathfrak{r}}_{t, n}=\sum_{m=1}^{M} \overline{\mathbf{s}}_{t, m} \bullet \overline{\mathfrak{h}}_{t, m, n}+\overline{\mathfrak{n}}_{t, n} .
$$

Recall that $\overline{\mathbf{s}}_{t, n}$ is the original modulated signal (before IFFT). 
Denote $\mathcal{H}_{\mathbf{t}}=\left\{\overline{\mathfrak{h}}_{t, m, n}\right\}_{M \times N}$ to be the matrix whose elements are the $N_{f f t}$-point FFTs of the respective elements in the channel coefficient matrix $\mathbf{H}$. Further, denote $\mathbf{R}=\left\{\overline{\mathbf{r}}_{O F D M, t, n}\right\}_{T \times N}$ to be the received signal matrix, $\mathcal{R}_{t}=\left\{\overline{\mathfrak{r}}_{t, n}\right\}_{T \times N}$ to be the received signal matrix after FFT, and $\mathcal{N}=\left\{\overline{\mathfrak{n}}_{t, n}\right\}_{T \times N}$ to be the noise matrix. We can rewrite (3) in matrix form as follows

$$
\mathcal{R}_{\mathbf{t}}=\mathbf{S}_{t} \circ \mathcal{H}_{\mathbf{t}}+\mathcal{N}_{\mathbf{t}}
$$

where we define the multiplication operation $\circ$ between $\mathbf{S}$ and $\mathcal{H}_{t}$ such that the $(t, n)$-th element of the resulting matrix is a $N_{f f t}$-length column vector $\sum_{m=1}^{M} \overline{\mathbf{s}}_{t, m} \bullet \overline{\mathfrak{h}}_{t, m, n}$. form

If we rewrite $\mathcal{R}_{\mathbf{t}}, \mathbf{S}_{t}, \mathcal{H}_{t}$ and $\mathcal{N}_{t}$ in (4) in the following

$$
\begin{aligned}
\mathbb{R}_{t} & =\left\{\operatorname{diag}\left(\overline{\mathfrak{r}}_{t, n}\right)\right\}_{T N_{f f t} \times N N_{f f t}}, \\
\mathbb{H}_{t} & =\left\{\operatorname{diag}\left(\overline{\mathfrak{h}}_{t, m, n}\right)\right\}_{M N_{f f t} \times N N_{f f t}}, \\
\mathbb{S}_{t} & =\left\{\operatorname{diag}\left(\overline{\mathbf{s}}_{t, m}\right)\right\}_{T N_{f f t} \times M N_{f f t}}, \\
\mathbb{N}_{t} & =\left\{\operatorname{diag}\left(\overline{\mathfrak{n}}_{t, n}\right)\right\}_{T N_{f f t} \times N N_{f f t}},
\end{aligned}
$$

then (4) can be rewritten with the normal definition of matrix multiplication as follows

$$
\mathbb{R}_{t}=\mathbb{S}_{t} \mathbb{H}_{t}+\mathbb{N}_{t}
$$

Channel coefficients are assumed to be known at the receiver to facilitate the coherent detection. In this case, due to the full orthogonality of STFCs [4], each MB-OFDM symbol $\overline{\mathbf{s}}_{t, m}$, which is a $\left(N_{f f t} \times 1\right)$-sized column vector, can be decoded separately. The decoding process is even more simplified by the fact that each element $s_{t, m, j}$ within $\overline{\mathbf{s}}_{t, m}$ can also be decoded separately. In the case of QOSTFCs, thanks to the partial orthogonality of QOSTFCs [7], [8], each pair of MBOFDM symbols each of which is a $\left(N_{f f t} \times 1\right)$-sized column vector can be decoded separately. Similarly, the decoding process is even more simplified by the fact that each pair of elements within the pair of MB-OFDM symbols can also be decoded separately. We refer readers to the publications [4], [7], [8] for more detail about the decoding metrics of STFCs and QOSTFCs in coherent detection.

\section{DIFFERENTIAL STFCS IN MB-OFDM UWB}

The proposed model of a DSTFC MB-OFDM UWB system requiring no transmission of channel estimation symbols is depicted in Fig. 2. As opposed to the STFC MB-OFDM system mentioned above where channels are normally assumed to be constant during multiple blocks of the transmitted STFC, in the DSTFC MB-OFDM system, it is required that channels must be constant during at least two consecutive blocks of the transmitted DSTFC. Thus the DSTFC MB-OFDM system is better fit for fast fading channels. Let us assume generally that channels in the DSTFC MB-OFDM system are constant during a time window ${ }^{1}$ of size $2 K T_{S Y M}$ (ns) where $K$ is an integer

\footnotetext{
${ }^{1}$ The time window in a DSTFC MB-OFDM system is smaller than that in a STFC MB-OFDM one because channels are constant during at least two consecutive code blocks in the former while they are constant during a larger number of code blocks in the latter.
}

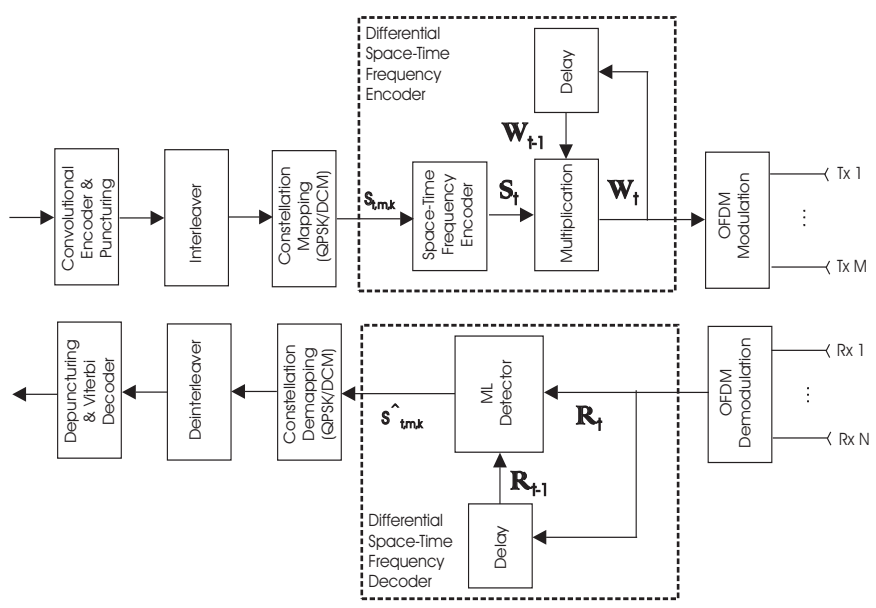

Fig. 2. Structural diagram of the proposed DSTFC MB-OFDM UWB system.

and $T_{S Y M}$ is the MB-OFDM symbol interval $T_{S Y M}=312.5$ ns [1].

We consider the application of the Alamouti STFC

$$
\mathbf{S}_{\mathbf{t}}=\overline{\mathbf{1}} / \sqrt{2} \circ\left[\begin{array}{rr}
\overline{\mathbf{s}}_{t, 1} & \overline{\mathbf{s}}_{t, 2} \\
-\overline{\mathbf{s}}_{t, 2}^{*} & \overline{\mathbf{s}}_{t, 1}^{*}
\end{array}\right],
$$

where the MB-OFDM symbol $\overline{\mathbf{s}}_{t, m}$, for $m=1,2$, is a column vector of $N_{f f t}$ data corresponding to $N_{f f t}$ sub-carriers, i.e. $\overline{\mathbf{s}}_{t, m}=\left[s_{t, m, 1} s_{t, m, 2} \ldots s_{t, m, N_{f f t}}\right]^{T}$. Further, we assume that the normalized power of each symbol $s_{t, m, k}$ within $\overline{\mathbf{s}}_{t, m}$, for $k=1, \ldots, N_{f f t}$, is unitary, i.e. $\left|s_{t, m, k}\right|^{2}=1$. Hence $s_{t, m, k}$ can be drawn from a PSK or 4QAM signal constellation.

The STFC in (6) can be rewritten in the following form

$$
\mathbb{S}_{t}=1 / \sqrt{2}\left[\begin{array}{rr}
\operatorname{diag}\left(\overline{\mathbf{s}}_{t, 1}\right) & \operatorname{diag}\left(\overline{\mathbf{s}}_{t, 2}\right) \\
-\operatorname{diag}\left(\overline{\mathbf{s}}_{t, 2}^{*}\right) & \operatorname{diag}\left(\overline{\mathbf{s}}_{t, 1}^{*}\right)
\end{array}\right] \text {. }
$$

Because the symbols $s_{t, m, k}$ are drawn from a unitary signal constellation, it is easy to realize that $\mathbb{S}_{t}$ is a unitary matrix of size $2 N_{f f t}$, i.e.

$$
\mathbb{S}_{t}^{H} \mathbb{S}_{t}=I_{2 N_{f f t}} .
$$

The proposed DSTFC MB-OFDM system initializes the transmission with an identity matrix $\mathbb{W}_{0}=I_{2 N_{f f t}}$. The following code matrices will be generated and transmitted according to the below principle

$$
\mathbb{W}_{t}=\mathbb{S}_{t} \mathbb{W}_{t-1}
$$

Similarly to $\mathbb{S}_{t}, \mathbb{W}_{t}$ can be represented in the form of column vectors, that are denoted as $\overline{\mathbf{w}}_{t, 1}$ and $\overline{\mathbf{w}}_{t, 2}$, i.e.

$$
\mathbf{W}_{\mathbf{t}}=\left[\begin{array}{rr}
\overline{\mathbf{w}}_{t, 1} & \overline{\mathbf{w}}_{t, 2} \\
-\overline{\mathbf{w}}_{t, 2}^{*} & \overline{\mathbf{w}}_{t, 1}^{*}
\end{array}\right] \text {, }
$$

As stated earlier in Section I, in a MB-OFDM system, consecutive MB-OFDM symbols are transmitted over different sub-bands. Thus, in the proposed DSTFC MB-OFDM system, the MB-OFDM symbols (the column vectors) in the same row of the matrix $\mathbf{W}_{\mathbf{t}}$, which will be transmitted over different $\mathrm{Tx}$ antennas in the same MB-OFDM symbol time slot, must be transmitted over the same sub-band. The MB-OFDM symbols 
in the same column of the matrix Wt, which will be transmitted over a certain Tx antenna during different MB-OFDM symbol time slots, can be instead transmitted over different sub-bands, according to different TFCs [1, p.60].

The assumption of channels being constant within a time window $2 K T_{S Y M}$ is equivalent to the fact that channels are constant during the transmission of $K$ consecutive Alamouti code blocks. Therefore, the encoding principle (9) should be applied for $t=1, \ldots,(K-1)$ and the whole transmission protocol is reset for a new time window. Since $\mathbb{S}_{t}$ is a unitary matrix, every transmitted code block $\mathbb{W}_{t}$ is also a unitary one, i.e. $\mathbb{W}_{t}^{H} \mathbb{W}_{t}=I_{2 N_{f f t}}$. The transmission model can be expressed as follows

$$
\mathbb{R}_{\mathbf{t}}=\mathbb{W}_{t} \mathbb{H}_{t}+\mathbb{N}_{t}
$$

We will now derive the maximum likelihood (ML) decoding metric for the proposed DSTFC. To do that, we first represent the STFC in (7) in the following form

$$
\mathbb{S}_{t}=1 / \sqrt{2} \sum_{m=1}^{2} \sum_{k=1}^{N_{f f t}}\left(\mathbb{X}_{t, m, k} s_{t, m, k}^{R}+i \mathbb{Y}_{t, m, k} s_{t, m, k}^{I}\right),
$$

where $s_{t, m, k}^{R}$ and $s_{t, m, k}^{I}$ are the real and imaginary parts of the symbol $s_{t, m, k}$ respectively, i.e. $s_{t, m, k}=s_{t, m, k}^{R}+i s_{t, m, k}^{I}$, while $\mathbb{X}_{t, m, k}$ and $\mathbb{Y}_{t, m, k}$ are their corresponding weighting matrices. We recall that the symbols $s_{t, m, k} \mathrm{~s}$ are drawn from the original PSK or 4QAM signal constellation.

The weighting matrices $\mathbb{X}_{t, m, k}$ and $\mathbb{Y}_{t, m, k}$ in the matrix (7) always satisfy the following properties for a given value $t$

$$
\begin{aligned}
\mathbb{X}_{t, m, k} \mathbb{X}_{t, m, k}^{H} & =\mathbb{I}, \mathbb{Y}_{t, m, k} \mathbb{Y}_{t, m, k}^{H}=\mathbb{I} \quad \forall m, k \\
\mathbb{X}_{t, m, k} \mathbb{X}_{t, \dot{m}, \dot{k}}^{H} & =-\mathbb{X}_{t, \dot{m}, \dot{k}} \mathbb{X}_{t, m, k}^{H}, \forall\{m, k\} \neq\{\dot{m}, \dot{k}\} \\
\mathbb{Y}_{t, m, k} \mathbb{Y}_{t, \dot{m}, \dot{k}}^{H} & =-\mathbb{Y}_{t, \dot{m}, \dot{k}} \mathbb{Y}_{t, m, k}^{H}, \forall\{m, k\} \neq\{\dot{m}, \dot{k}\} \\
\mathbb{X}_{t, m, k} \mathbb{Y}_{t, \dot{m}, \dot{k}}^{H} & =\mathbb{Y}_{t, \dot{m}, \dot{k}} \mathbb{X}_{t, m, k}^{H} \quad \forall m, k, \dot{m}, \dot{k}
\end{aligned}
$$

To formulate the ML decoding metric for the symbol $s_{t, m, k}$, for $t=1, \ldots,(K-1), m=1,2$ and $k=1, \ldots, N_{f f t}$, let us consider the following term

$$
D_{m, k}=D_{m, k}^{R}+i D_{m, k}^{I},
$$

where

$$
\begin{aligned}
D_{m, k}^{R} & =\Re\left[\operatorname{tr}\left(\mathbb{R}_{t}^{H} \mathbb{R}_{t-1} \mathbb{X}_{t, m, k}\right)\right] \\
D_{m, k}^{I} & =\Re\left[\operatorname{tr}\left(\mathbb{R}_{t}^{H} \mathbb{R}_{t-1} i \mathbb{Y}_{t, m, k}\right)\right]
\end{aligned}
$$

We have

$$
\begin{aligned}
D_{m, k}^{R} & =\Re\left[\operatorname{tr}\left(\mathbb{R}_{t}^{H} \mathbb{R}_{t-1} \mathbb{X}_{t, m, k}\right)\right] \\
& =\Re\left\{\operatorname{tr}\left[\left(\mathbb{W}_{t} \mathbb{H}_{t}+\mathbb{N}_{t}\right)^{H}\left(\mathbb{W}_{t-1} \mathbb{H}_{t-1}+\mathbb{N}_{t-1}\right) \mathbb{X}_{t, m, k}\right]\right\} \\
& =\Re\left\{\operatorname{tr}\left[\left(\mathbb{S}_{t}^{H}\left(\mathbb{H}_{t}^{H} \mathbb{H}_{t-1}\right)\left(\mathbb{W}_{t-1}^{H} \mathbb{W}_{t-1}\right)+\mathbb{N}\right) \mathbb{X}_{t, m, k}\right]\right\}
\end{aligned}
$$

where

$$
\mathbb{N}:=\mathbb{H}_{t}^{H} \mathbb{W}_{t}^{H} \mathbb{N}_{t-1}+\mathbb{N}_{t}^{H} \mathbb{W}_{t-1} \mathbb{H}_{t-1}+\mathbb{N}_{t}^{H} \mathbb{N}_{t-1}
$$

Because $\mathbb{W}_{t-1}$ is a unitary matrix, i.e.

$$
\mathbb{W}_{t-1}^{H} \mathbb{W}_{t-1}=I_{2 N_{f f t}}
$$

and channel coefficients are constant during a time window of $K$ code blocks, i.e. $\mathbb{H}_{t}=\mathbb{H}_{t-1}$, Eq. (16) becomes

$$
D_{m, k}^{R}=\Re\left\{\operatorname{tr}\left[\left(\mathbb{H}_{t}^{H} \mathbb{H}_{t}\right)\left(\mathbb{S}_{t}^{H} \mathbb{X}_{t, m, k}\right)\right]\right\}+\Re\left\{\operatorname{tr}\left(\mathbb{N X}_{t, m, k}\right)\right\} .
$$

The first term is calculated as follows

$$
\begin{aligned}
& \Re\left\{\operatorname{tr}\left(\mathbb{H}_{t}^{H} \mathbb{H}_{t} \mathbb{S}_{t}^{H} \mathbb{X}_{t, m, k}\right)\right\}== \\
& 1 / \sqrt{2} \Re\left\{\operatorname { t r } \left[\mathbb { H } _ { t } ^ { H } \mathbb { H } _ { t } \left(\mathbb{X}_{t, m, k}^{H} \mathbb{X}_{t, m, k} s_{t, m, k}^{R}\right.\right.\right. \\
&+\sum_{\forall \dot{m}, \dot{k},\{\dot{m}, \hat{k}\} \neq\{m, k\}} \mathbb{X}_{t, \dot{m}, \dot{k}}^{H} \mathbb{X}_{t, m, k} s_{t, \dot{m}, \dot{k}}^{R} \\
&\left.\left.\left.+i \sum_{\forall \dot{m}, \dot{k}} \mathbb{Y}_{t, \dot{m}, \dot{k}}^{H} \mathbb{X}_{t, m, k} s_{t, \dot{m}, \dot{k}}^{I}\right)\right]\right\} .
\end{aligned}
$$

It is noted that if $\Phi$ is an antihermitian (or skew-Hermitian) matrix, i.e. $\Phi^{H}=-\Phi$, then $\operatorname{tr}\left(\mathbb{A}^{H} \mathbb{A} \Phi\right)$ is imaginary, thus $\Re\left\{\operatorname{tr}\left(\mathbb{A}^{H} \mathbb{A} \Phi\right)\right\}=0$. From (13), it is easy to check that $\left(\mathbb{X}_{t, \dot{m}, \dot{k}}^{H} \mathbb{X}_{t, m, k} s_{t, \dot{m}, \dot{k}}^{R}\right)^{H}=-\left(\mathbb{X}_{t, \dot{m}, \dot{k}}^{H} \mathbb{X}_{t, m, k} s_{t, \dot{m}, \dot{k}}^{R}\right)$, i.e. $\left(\mathbb{X}_{t, \dot{m}, \dot{k}}^{H} \mathbb{X}_{t, m, k} s_{t, \dot{m}, \dot{k}}^{R}\right)$ is an antihermitian matrix, thus

$$
\left.\Re\left\{\operatorname{tr}\left(\mathbb{H}_{t}^{H} \mathbb{H}_{t} \mathbb{X}_{t, \dot{m}, \dot{k}}^{H} \mathbb{X}_{t, m, k} s_{t, \dot{m}, \dot{k}}^{R}\right)\right)\right\}=0,
$$

for all $\dot{m}, \hat{k}$ and $\{\dot{m}, \hat{k}\} \neq\{m, k\}$.

On the other hand, if $\Theta$ is a Hermitian matrix, i.e. $\Theta^{H}=\Theta$, then $\operatorname{tr}\left(\mathbb{A}^{H} \mathbb{A} \Theta\right)$ is real, thus $\Im\left\{\operatorname{tr}\left(\mathbb{A}^{H} \mathbb{A} \Theta\right)\right\}=0$. From (15), it is trivial to realize that $\left(\mathbb{Y}_{t, m, \dot{k}}^{H} \mathbb{X}_{t, m, k} s_{t, k}^{I}\right)^{H}=$ $\left(\mathbb{Y}_{t, \dot{m}, \hat{k}^{H}}^{H} \mathbb{X}_{t, m, k} s_{t, k}^{I}\right)$, i.e. $\left(\mathbb{Y}_{t, \dot{m}, \hat{k}^{H}}^{H} \mathbb{X}_{t, m, k} s_{t, k}^{I}\right)$ is a Hermitian matrix, thus

$$
\begin{aligned}
\Re\left\{\operatorname{tr}\left(\mathbb{H}_{t}^{H} \mathbb{H}_{t} \mathbb{Y}_{t, \dot{m}, \dot{k}}^{H} i \mathbb{X}_{t, m, k} s_{t, \dot{m}, \dot{k}}^{I}\right)\right\} \\
\quad=\Im\left\{\operatorname{tr}\left(\mathbb{H}_{t}^{H} \mathbb{H}_{t} \mathbb{Y}_{t, \dot{m}, \dot{k}}^{H} \mathbb{X}_{t, m, k} s_{t, \dot{m}, \dot{k}}^{I}\right)\right\} \\
=0 \quad \forall m, k, \dot{m}, \hat{k}
\end{aligned}
$$

If we denote $\mathbb{C}_{t, m, k}:=\mathbb{H}_{t} \mathbb{H}_{t}^{H} \mathbb{X}_{t, m, k}^{H} \mathbb{X}_{t, m, k}$ then $\mathbb{C}_{t, m, k}$ is a constant matrix for given values $t, m$ and $k$ and $\operatorname{tr}\left(\mathbb{C}_{t, m, k}\right)$ is a positive real number (the trivial case $\operatorname{tr}\left(\mathbb{C}_{t, m, k}\right)=0$ is discarded). From (17)-(20), we have

$$
D_{m, k}^{R}=1 / \sqrt{2} \Re\left\{\operatorname{tr}\left(\mathbb{H}_{t}^{H} \mathbb{H}_{t}\right)\right\} s_{t, m, k}^{R}+\Re\left\{\operatorname{tr}\left(\mathbb{N X}_{t, m, k}\right)\right\}
$$

The term $D_{m, k}^{I}$ is calculated in a similar way with the note that $\mathbb{H}_{t} \mathbb{H}_{t}^{H} \mathbb{Y}_{t, m, k} \mathbb{Y}_{t, m, k}^{H}=\mathbb{H}_{t} \mathbb{H}_{t}^{H} \mathbb{X}_{t, m, k} \mathbb{X}_{t, m, k}^{H}=\mathbb{C}_{t, m, k}$ (cf. Eq. (12)), we have

$$
D_{m, k}^{I}=1 / \sqrt{2} \operatorname{tr}\left(\mathbb{C}_{t, m, k}\right) s_{t, m, k}^{I}+\Re\left\{\operatorname{tr}\left(\mathbb{N}_{i} \mathbb{Y}_{t, m, k}\right)\right\} .
$$

Therefore

$$
\begin{aligned}
D_{m, k} & =D_{m, k}^{R}+i D_{m, k}^{I} \\
& =1 / \sqrt{2} \operatorname{tr}\left(\mathbb{C}_{t, m, k}\right) s_{t, m, k} \\
& +\Re\left\{\operatorname{tr}\left(\mathbb{N X}_{t, m, k}\right)\right\}+i \Re\left\{\operatorname{tr}\left(\mathbb{N}_{i} \mathbb{Y}_{t, m, k}\right)\right\} .
\end{aligned}
$$


TABLE I

SIMULATION PARAMETERS.

\begin{tabular}{l|r}
\hline Parameter & Value \\
\hline FFT and IFFT size & $N_{f f t}=128$ \\
Data rate & $320 \mathrm{Mbps}$ \\
Convolutional encoder's rate & $1 / 2$ \\
Convolutional encoder's constraint length & 7 \\
Convolutional decoder & Viterbi \\
Decoding mode & Hard \\
STFC decoding at nodes & ML decoding \\
Number of transmitted & 1200 \\
DSTFC blocks & Unitary QPSK \\
Modulation & CM1, $2,3 \& 4$ \\
IEEE Channel model & $N_{D}=100$ \\
Number of data subcarriers & $N_{P}=12$ \\
Number of pilot subcarriers & $N_{G}=10$ \\
Number of guard subcarriers & $N_{T}=122$ \\
Total number of subcarriers used & $N_{Z P S}=37$ \\
Number of samples in ZPS & $N_{S Y M}=165$ \\
Total number of samples/symbol & 100 \\
Number of channel realizations & \\
\hline
\end{tabular}

The ML decoding metric for $s_{t, m, k}$ can be derived as follows

$$
\begin{aligned}
\hat{s}_{t, m, k} & =\arg \min \left|D_{m, k}-\frac{1}{\sqrt{2}} \operatorname{tr}\left(\mathbb{C}_{t, m, k}\right) s_{t, m, k}\right|^{2} \\
& =\arg \min \left(\left|D_{m, k}\right|^{2}+\frac{1}{2}\left[\operatorname{tr}\left(\mathbb{C}_{t, m, k}\right)\right]^{2}\left|s_{t, m, k}\right|^{2}\right. \\
& \left.-\sqrt{2} \operatorname{tr}\left(\mathbb{C}_{t, m, k}\right) \Re\left\{D_{m, k}^{*} s_{t, m, k}\right\}\right) \\
& =\arg \min \left(\left|D_{m, k}\right|^{2}+\frac{1}{2}\left[\operatorname{tr}\left(\mathbb{C}_{t, m, k}\right)\right]^{2}\right. \\
& \left.-\sqrt{2} \operatorname{tr}\left(\mathbb{C}_{t, m, k}\right) \Re\left\{D_{m, k}^{*} s_{t, m, k}\right\}\right) .
\end{aligned}
$$

Since $\mathbb{C}_{t, m, k}$ is a constant matrix for given $t, m$ and $k$ and because $\operatorname{tr}\left(\mathbb{C}_{t, m, k}\right)$ is a positive real number, the equivalent ML decoding metric for $s_{t, m, k}$ is

$$
\hat{s}_{t, m, k}=\arg \max _{s_{t, m, k} \in \mathcal{C}}\left(\Re\left\{D_{m, k}^{*} s_{t, m, k}\right\}\right),
$$

for $t=1, \ldots,(K-1), m=1,2$ and $k=1, \ldots, N_{f f t}$. In fact, there are only $N_{D}=100$ data symbols within each MBOFDM symbol, that include 28 other pilot, guard and null symbols [1]. Therefore, instead of decoding $N_{f f t}$ symbols in Eq. (21), i.e. $k=1, \ldots, N_{f f t}$, we only need to decode $N_{D}$ symbols, i.e. $k=1, \ldots, N_{D}$.

Eq. (21) means that each of the two MB-OFDM symbols $\overline{\mathbf{s}}_{\mathbf{t}, \mathbf{1}}$ and $\overline{\mathbf{s}}_{\mathbf{t}, \mathbf{2}}$ can be separately decoded. Furthermore, each symbol $s_{t, m, k}$ within these two MB-OFDM symbols can also be separately decoded based on the above equation. In other words, instead of jointly decoding all $2 N_{f f t}$ symbols $s_{t, m, k}$ within the two MB-OFDM symbols $\overline{\mathbf{s}}_{\mathbf{t}, \mathbf{1}}$ and $\overline{\mathbf{s}}_{\mathbf{t}, \mathbf{2}}$ at a time, each of them can be separately decoded. No CSI is required for the decoding process. All we need for the decoding process at time $t$ are the received signals at the previous time $(t-1)$ and at the current time. The decoding process is completely linear, thus relatively simple.

\section{Simulation Results}

To examine the performance advantage of the proposed DSTFC MB-OFDM system, several Monte-Carlo simulations were run in Matlab ${ }^{T M}$ for the baseband, conventional differential MB-OFDM system (without MIMO) and the baseband
Alamouti DSTFC MB-OFDM system at the bit rate 320 Mbps. The conventional differential MB-OFDM system was simply created in a similar way as Eq. (9), but it was much more simplified because transmission was carried out in a symbol-by-symbol basis rather than a block-by-block basis in a DSTFC MB-OFDM system. In particular, the transmitted MB-OFDM symbol at time $t$ is the Hadamard product of the transmitted symbol at time $t-1$ and an information symbol. The initial transmitted symbol is set to the vector $\overline{\mathbf{1}}$. Decoding process was also carried out in a similar manner to Eq. (21).

Each run of simulations was carried out with 1200 Alamouti DSTFC blocks. One hundred channel realizations of each channel model (CM 1 to $\mathrm{CM} 4$ ) were considered for the transmission of each DSTFC block. The channel realizations were created by the Matlab program enclosed in the appendix of the IEEE 802.15.3a channel modeling sub-committee report [18]. Channel coefficients were assumed to be constant during every two consecutive transmissions of DSTFC blocks. In simulations, $S N R$ is defined to be the signal-to-noise ratio (dB) per sample in a MB-OFDM symbol (consisting of 165 samples), at each Rx antenna (i.e. the subtraction between the total power $(\mathrm{dB})$ of the received signal corresponding to the sample of interest and the power of noise $(\mathrm{dB})$ at that $\mathrm{Rx}$ antenna). In both systems, symbols $s_{t, m, k}$ were drawn from unitary QPSK signal constellations, i.e. $\left|s_{t, m, k}\right|^{2}=1$. This condition, along with the unitary structure of the transmitted DSTFC blocks, guarantees the power constraint, i.e. the total powers transmitted from all Tx antennas are equal in both systems at any time, in order to fairly compare their performance. The complete set of simulation parameters is presented in Table I.

Fig. 3 compares the bit error performance of the two systems in the case where the receiver is equipped with only one Rx antenna. From this figure, the proposed DSTFC system brings about a significant improvement in the bit error performance, compared to the conventional differential MBOFDM system. For instance, the Alamouti DSTFC provides approximately a $4 \mathrm{~dB}$ gain in the case of CM 1 at the bit error rate $B E R=10^{-3}$. The more dispersive the channel is (CM 1 is the least dispersive channel model while CM 4 is the most dispersive one), the higher gain the DSTFC provides.

Fig. 4 presents the bit error performance of the two systems in the case of two Rx antennas. Once again, we can see that DSTFCs improve significantly the bit error performance of MB-OFDM systems. For illustration, a gain of at least $6 \mathrm{~dB}$ (over the conventional differential MB-OFDM system) can be achieved at $B E R=10^{-5}$ when the Alamouti DSTFC is utilized. It is noted that the aforementioned improvements were gained without any increase of total transmission power.

\section{Conclusions}

The paper has proposed the framework of DSTFC MBOFDM UWB systems using the unitary Alamouti DSTFC. It has been shown that MIMO MB-OFDM systems using DSTFCs can possess much better bit error performance, compared to the conventional differential MB-OFDM UWB without MIMO. Furthermore, beside the case study of DSTFC MBOFDM UWB, it is our conjecture that the proposed DSTFC 


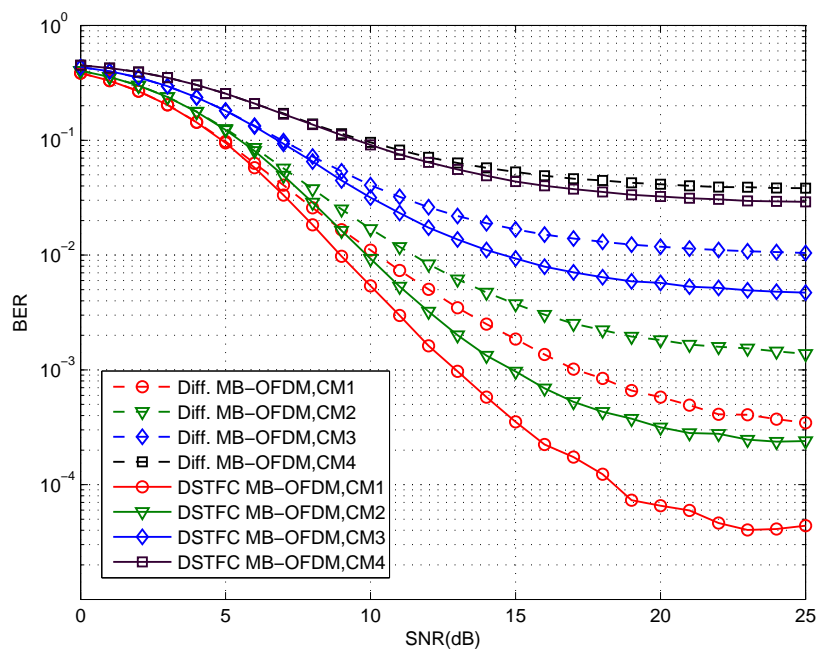

Fig. 3. DSTFC MB-OFDM vs. differential MB-OFDM (without STFCs) in the case of one Rx antenna.

principle might be applied in various other wireless applications, such as WiMax MIMO [24], providing better $B E R$ than the respective WiMax without MIMO. Examination of the application of DSTFCs in WiMax MIMO will be our future work.

\section{ACKNOWLEDGMENT}

L. C. Tran would like to thank the Alexander von Humboldt (AvH) Foundation, Germany, for its support of this work under the form of a postdoctoral fellowship.

\section{REFERENCES}

[1] A. Batra et al., "Multiband OFDM physical layer specification.," WiMedia Alliance Release 1.1, July 2005.

[2] T.-H. Tan and K.-C. Lin, "Performance of space-time block coded MBOFDM UWB systems," Proc. 4th Annual Communication Networks and Services Research Conference (CNSR'06), pp. 323 - 327, May 2006.

[3] W. P. Siriwongpairat, W. Su, M. Olfat, and K. J. R. Liu, "MultibandOFDM MIMO coding framework for UWB communication systems," IEEE Trans. Sign. Process., vol. 54, no. 1, pp. 214 - 224, Jan. 2006.

[4] L. C. Tran and A. Mertins, "Space-time frequency code implementation in MB-OFDM UWB communications: design criteria and performance," IEEE Trans. Wireless Commun., vol. 8, no. 2, pp. 701-713, Feb. 2009

[5] L. C. Tran, A. Mertins, E. Dutkiewicz, and X. Huang, "Space-timefrequency codes in MB-OFDM UWB communications: Advanced order8 STFC and its performance," Proc. 7th IEEE International Symposium on Communications and Information Technologies ISCIT 2007, Oct. 2007.

[6] L. C. Tran, A. Mertins, and T. A. Wysocki, "Quasi-orthogonal spacetime-frequency codes in MB-OFDM UWB communications," Proc. International Conference on Signal Processing and Communication Systems ICSPCS2007, Dec. 2007.

[7] L. C. Tran and A. Mertins, "On the use of quasi-orthogonal space-timefrequency codes in MB-OFDM UWB," Proc. 2nd IEEE International Conference on Communication and Electronics (ICCE2008), pp. 252257, June 2008.

[8] L. C. Tran and A. Mertins, "Application of quasi-orthogonal spacetime-frequency codes in MB-OFDM UWB," Proc. IEEE International Conference on Ultra-Wideband (ICUWB 2008)., Sept. 2008.

[9] Q. Yang and K. S. Kwak, "Superimposed training for estimating of doubly-selective OFDM channels," Proc. 9th Int. Conf. Advanced Communication Technology (ICACT 2007), vol. 3, pp. 1652-1655, Feb. 2007.

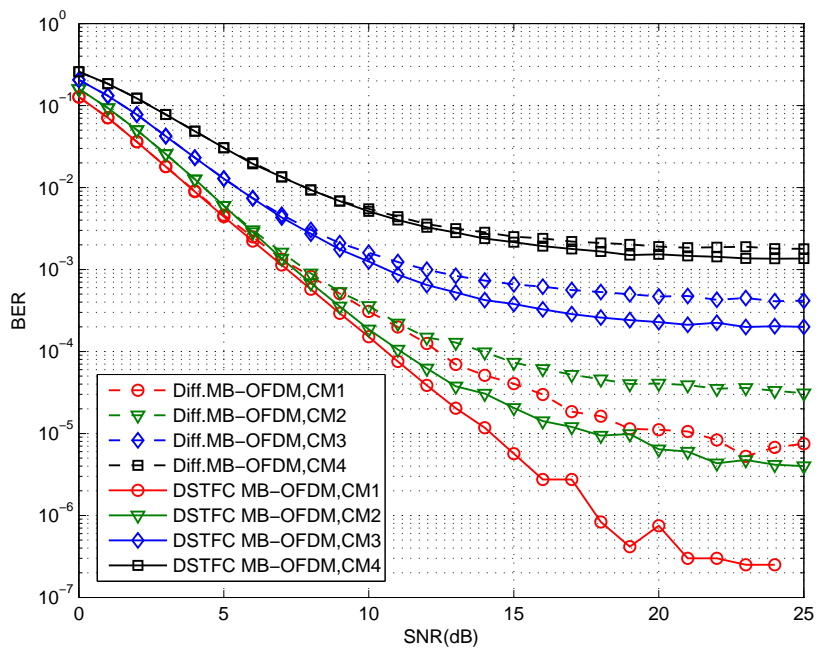

Fig. 4. DSTFC MB-OFDM vs. differential MB-OFDM (without STFCs) in the case of two Rx antennas.

[10] K. Josiam and D. Rajan, "Bandwidth efficient channel estimation using super-imposed pilots in OFDM systems," IEEE Trans. Wireless Commun., vol. 6, pp. 2234-2245, June 2007.

[11] S. Lu, G. Kang, Q. Zhu, and P. Zhang, "A orthogonal superimposed pilot for channel estimation in MIMO-OFDM systems," Proc. IEEE 65th Vehicular Technology Conference (VTC2007-Spring), pp. 24092413, Apr. 2007.

[12] H. Li, "Differential space-time-frequency modulation over frequencyselective fading channels," IEEE Commun. Letter, vol. 7, no. 8, pp. 349-351, Aug. 2003.

[13] J. Wang and K. Yao, "Differential unitary space-time-frequency coding for MIMO OFDM systems," Proc. IEEE 36-th Asilomar Conference on Signals, Systems and Computers, vol. 2, pp. 1867-1871, Nov. 2002.

[14] Q. Ma, C. Tepedelenlioglu, and Z. Liu, "Differential spacetimefrequency coded OFDM with maximum multipath diversity," IEEE Trans. Wireless Commun., vol. 4, no. 5, pp. 2232-2243, Sept. 2005.

[15] S. N. Diggavi, N. Al-Dhahir, A. Stamoulis, and A. R. Calderbank, "Differential space-time coding for frequency-selective channels," IEEE Commun. Letter, vol. 6, no. 6, pp. 253-255, June 2002.

[16] T. Himsoon, W. Su, and K. J. R. Liu, "Single-block differential transmit scheme for broadband wireless mimo-ofdm systems," IEEE Trans. Signal Process., vol. 54, no. 9, pp. 3305-3314, Sept. 2006.

[17] Z. Liu and G. B. Giannakis, "Block differentially encoded OFDM with maximum multipath diversity," IEEE Trans. Wireless Commun., vol. 2, no. 3, pp. 420-423, May 2003.

[18] J. Foerster and Intel R\&D, "Channel modelling sub-committee report final," IEEE P802.15 Working Group for Wireless Personal Area Networks (WPANs), IEEE P802.15-02/490r1-SG3a, Oct. 2005.

[19] S. M. Alamouti, "A simple transmit diversity technique for wireless communications," IEEE J. Select. Areas Commun., vol. 16, no. 8, pp. 1451 - 1458, Oct. 1998.

[20] L. C. Tran, T. A. Wysocki, A. Mertins, and J. Seberry, Complex Orthogonal Space-Time Processing in Wireless Communications, Springer, New York, USA, 2006.

[21] V. Tarokh, H. Jafarkhani, and A. R. Calderbank, "Space-time blocks codes from orthogonal designs," IEEE Trans. Inform. Theory, vol. 45, no. 5, pp. 1456 - 1467, July 1999 .

[22] H. Jafarkhani, "A quasi-orthogonal space-time block codes," IEEE Trans. Commun., vol. 49, no. 1, pp. 1 - 4, Jan. 2001.

[23] A. Batra, J. Balakrishnan, A. Dabak, and many others, "Multi-band OFDM physical layer proposal for IEEE 802.15 task group 3a," IEEE P802.15-04/0493rl, Sept. 2004.

[24] R. B. Marks et al., "IEEE tandard for local and metropolitan area networks. Part 16: Air interface for fixed and mobile broadband wireless access systems," IEEE 802.16e-2005, Dec. 2005. 